\title{
Introduction of Basic Life Support in Medical Curriculum - Is this the Need of an Hour?
}

\author{
Radhika Rani Chandra1, Sunil Kumar Kilari², Sree Lakshmi Uppalapati , Anil Kumar Bathula4, \\ Muneer Kanha Mopuri' ${ }^{5}$, Shoba Katragadda 6
}

\begin{abstract}
${ }^{1}$ Assistant Professor, Department of Emergency Medicine, ACSR Government Medical College, Nellore, Andhra Pradesh, India and Faculty, AHA Dr. NTRUHS ITC, Vijayawada, Andhra Pradesh, India. ${ }^{2}$ Professor, Department of Nephrology, Katuri Medical

College, Guntur, Andhra Pradesh, India and Faculty, AHA Dr. NTRUHS ITC, Vijayawada, Andhra Pradesh, India. ${ }^{3}$ HOD, Department of Anaesthesia \& Critical Care, Nagarjuna Hospital, Vijayawada, Andhra Pradesh, India and Instructor, AHA Dr. NTRUHS ITC, Vijayawada, Andhra Pradesh, India. ${ }^{4}$ Assistant Professor, Department of SPM, Guntur Medical College, Guntur, Andhra Pradesh, India. ${ }^{5}$ Assistant Professor, Department of Pharmacology, Guntur Medical College, Guntur, Andhra Pradesh, India and Instructor, AHA Dr. NTRUHS ITC, Vijayawada, Andhra Pradesh, India. ${ }^{6}$ Academic Coordinator, AHA Dr. NTRUHS

ITC, Vijayawada, Andhra Pradesh, India.
\end{abstract}

\section{ABSTRACT}

\section{BACKGROUND}

Deaths do occur; but sometimes they can be prevented by adopting simple manoeuvres and skills. BCLS is a key component of chain of survival. The present study highlights the need for a structured training in BCLS and introduction of BCLS in the academic curriculum of undergraduate medical course. We wanted to measure the knowledge levels regarding Circulation Airway Breathing (CAB) Resuscitation in study subjects and assess the awareness of study subjects with regard to BCLS life supports.

\section{METHODS}

This is a cross sectional study conducted in Guntur Medical College and Hospital, Guntur, Andhra Pradesh, among 213 final year Part 1 \& 2 undergraduate students of medicine using a semi-structured questionnaire which is pre-tested, in the month of January 2019. The data was collected and entered in MS Excel and analysed by Epi Info.

\section{RESULTS}

Of the 213 study subjects, 159 (74.6\%) were females while 54 (25.3\%) were males. Out of the study subjects, $79.8 \%$ were aware of Basic Life Support; 77\% were having poor theoretical knowledge about Basic Life Support, among them, in $42.3 \%$ there was lack of knowledge with regard to BLS due to lack of professional training in their undergraduation. $97.18 \%$ think that Basic Life Support training should be a part of academic curriculum in medicine.

\section{CONCLUSIONS}

Basic Cardiopulmonary Life Support training is an absolute necessity for the medical students to face acute medical emergencies that they come across, even while pursuing their graduation. This also boosts the confidence of students.

\section{KEY WORDS}

Basic Life Support (BLS), Basic Cardiopulmonary Life Support (BCLS), Knowledge, Awareness, Medical Students, CPR (Cardiopulmonary Resuscitation), Circulation Airway Breathing (CAB), AED (Automated External Defibrillator)
Corresponding Author: Dr. Radhika Rani Chandra Department of Anaesthesia, Flat No. 202, 3rd Floor, Vijaymohan Residency, 6/1, Kannavarithota, Guntur-522004, Andhra Pradesh, India. E-mail: chandraradhikarani@gmail.com

DOI: $10.14260 / j e m d s / 2019 / 648$

Financial or Other Competing Interests: None.

How to Cite This Article: Chandra RR, Kilari SK, Uppalapati SL, et al. Introduction of basic life support in medical curriculum- is this the need of an hour?. J. Evolution Med. Dent. Sci. 2019;8(39):2982-2985, DOI: $10.14260 /$ jemds/2019/648

Submission 01-07-2019, Peer Review 10-09-2019, Acceptance 16-09-2019, Published 30-09-2019. 


\section{BACKGROUND}

Resuscitation "is the art of restoring life or consciousness of one apparently dead." [1] CPR invented in 1960, is a simple but effective procedure that allows almost anyone to sustain life in early critical minutes after cardiac or respiratory arrest. [2]

Basic Cardiopulmonary Life Support (BCLS) and Comprehensive Cardiac Life Support (CCLS) are part of CPR. ${ }^{[3,4]}$ Basic Cardiopulmonary Life Support (BCLS) includes early recognition of sudden cardiac arrest (SCA), acute coronary syndrome, stroke and foreign-body airway obstruction (FBAO), cardiopulmonary resuscitation (CPR), and defibrillation with an automated external defibrillator (AED).[5]

Deaths do occur; but sometimes it can be prevented by adopting simple manoeuvres and skills as it can prevent grave consequences and can increase the rate of hospital discharge. Every citizen in the community should know about Hands only CPR or Compression only life support as they are the first responders who can save lives and improve the quality of health in the community. Medical fraternity like doctors, nurses, paramedics should know about BCLS as they are the first responders with in the and out of the hospital life threatening conditions and knowledge in BCLS will be great help for them.

Basic Cardiopulmonary Life Support (BCLS) is a key component of chain of survival. The present study highlights the need for a structured training in BCLS and introduction of BCLS in the academic curriculum of medicine.

DRSCABD stands for

- D Dangers?

- R Responsive?

- $\quad$ S Send for help.

- C Start CPR.

- An Open Airway.

- B Normal breathing?

- D Attach Defibrillator.

We wanted to measure the knowledge levels regarding Circulation Airway Breathing (CAB)/Resuscitation in study subjects and to assess the awareness of study subjects in Basic cardiopulmonary Life Support.

\section{METHODS}

This is a cross sectional study conducted in Guntur Medical College and Hospital, Guntur, Andhra Pradesh among 213 undergraduate medical students in the month of January 2019 after institutional ethical committee approval. After taking verbal consent, 213 final year part 1 \& 2 undergraduate medical students who has given willingness to participate in the study were included. As levels of awareness and knowledge vary after clinical exposure from first year to final year students, final year medical students were included in the study. The data was collected using pre-tested, semistructured questionnaire and was distributed to the subjects and collected after adequate time.

A question bank comprising of 25 questions about the skills, knowledge, awareness in BCLS was used to assess their level of knowledge and practicality. They were questioned on their knowledge about BCLS, abbreviations of AED, CPR, BCLS, sequential assessment and resuscitation techniques of BCLS, their ability to recognize early signs of stroke and acute coronary syndrome, removal of foreign body obstruction. After excluding the incomplete response sheets the data was analysed on 213 responders. Permission was taken from the institutional head before involving the students. The results were analysed using an answer, key prepared from the Basic cardiopulmonary life support manual.

\section{Statistical Analysis}

Data collected was entered in MS Excel sheet by calculating appropriate proportions by using EPI INFO statistical software package.

\section{RESULTS}

Among 213 study subjects, 159(74.6\%) were females while $54(25.3 \%)$ were males. Out of study subjects, $79.8 \%$ were aware of Basic Cardiopulmonary Life Support (BCLS), 20.2\% medical students never heard about BCLS. $69 \%$ percent has never seen BCLS performed. $94.4 \%$ has never done BCLS on patients. $87.3 \%$ has never attended workshop on BCLS. 93.4\% do not think that BCLS should be done only in hospital. $82.2 \%$ never called emergency number. $38.8 \%$ never heard of Heimlich manoeuvre (Table 1).

Of the 213 study subjects, 48\% doesn't know the abbreviation of BCLS as Basic Cardiopulmonary Life Support. $83 \%$ failed to insist on scene safety as the first step in BCLS. $79 \%$ failed to insist on calling for help and activating the Emergency response system after confirmation of unresponsiveness of the victim. 84\% did not know the exact location of placement of their hands to do chest compressions which is two fingers above the xiphisternum. 83\% doesn't know that ventilation can be given with face mask, face shield and bag mask other than mouth to mouth ventilation. 33\% gave correct answer for rate of cardiac compression as $120 / \mathrm{min}$ in all subjects like adult, paediatric and infants. $72 \%$ doesn't know what AED stands for ('Automated External Defibrillator'). 84\% couldn't recognize the first signs of foreign body aspiration and obstruction and its severity. $10 \%$ only knew about the importance of recovery position in unresponsive victims who is breathing spontaneously. $66 \%$ did not know the Canadian stroke assessment scale for recognizing early signs of stroke and 58\% only know the signs of acute coronary syndrome. Seventy seven percent are having a poor theoretical knowledge about Basic Life Support, among them, $42.3 \%$ mean that their lack of knowledge in BLS is due to no awareness and no available professional training in their undergraduation and $97.18 \%$ think that Basic Cardiopulmonary Life Support training should be inculcated in their medical curriculum. Fifty percent think that Emergency physicians are the best qualified to guide them in BCLS training. (Figure 2, 3, 4). No one had complete knowledge on BCLS. Only Four out of 213 had secured $81-90 \%$ marks. Fifteen out of 213 had got 71 $80 \%$ marks. Thirty eight of 213 had got $61-70 \%$ marks. Sixty two of the 213 had secured $51-60 \%$ marks. The remaining ninety-four secured less than $50 \%$ marks. 


\begin{tabular}{|c|c|c|c|}
\hline Sl. No. & Question & Yes & No \\
\hline 1 & Heard about BLS? & $170(79.8 \%)$ & $43(20.2 \%)$ \\
\hline 2 & Seen BLS being done? & $66(31 \%)$ & $147(69 \%)$ \\
\hline 3 & Done BLS on patient? & $12(5.6 \%)$ & $201(94.4 \%)$ \\
\hline 4 & Attended any workshop on BLS? & $27(12.7 \%)$ & $186(87.3 \%)$ \\
\hline 5 & Think BLS should be done only in hospital? & $14(6.6 \%)$ & $199(93.4 \%)$ \\
\hline 6 & Called emergency number? & $38(17.8 \%)$ & $175(82.2 \%)$ \\
\hline 7 & Heard of Heimlich manoeuvre? & $131(61.1 \%)$ & $82(38.8 \%)$ \\
\hline \multicolumn{3}{|c|}{ Table 1. Awareness Regarding BLS } \\
\hline
\end{tabular}

\begin{tabular}{|c|c|}
\hline $2.8 \%$ & $\begin{array}{l}\text { Figure } 1 . \\
\text { Think BLS Training should be a } \\
\text { Part of Medical Curriculum? }\end{array}$ \\
\hline
\end{tabular}
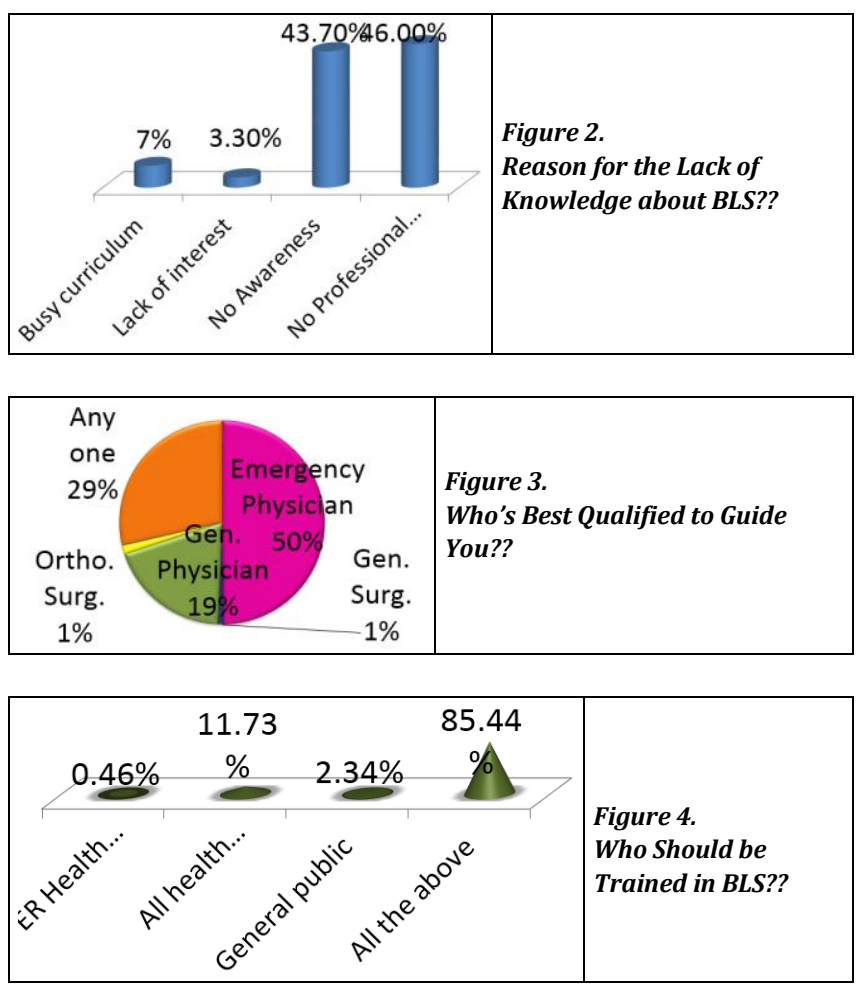

\section{DISCUSSION}

The study results showed that medical students in the study group were severely lacking in the awareness of BCLS and also emphasizes the cognitive approach to the general awareness, perception and skills of Basic life support, early recognition of Acute Coronary Syndrome and Stroke.[6],[7]

Ruesseler $\mathrm{M}$ et al also mentioned in their study that simulation training improves ability to manage medical emergencies.[8] Shanta Chandrasekaran et al reported awareness of basic life support among medical, dental, nursing students and found only 2 out of 1054 had secured $80-90 \%$ marks. In our study, only 4 out of 213 secured 80 90\% marks in the test. ${ }^{[9]}$ Hassan Zaheer et al concluded that inclusion of BLS course will increase awareness and application of this valuable lifesaving manoeuvre.[10] Therefore, BLS training programmes should be mandatory for all medical students.

C. A. Graham et al studied a survey of undergraduate training in UK medical schools and found similar results.[11]
Asad abbas et al and Pakistan journal showed that knowledge of trained student was found to be better than untrained student. ${ }^{[12]}$ S. Pande et al concluded a lack of awareness regarding BLS among medical students and suggested that resuscitation skills become a part of the undergraduate curriculum and after the BLS training, student knowledge and skills improved, and there was a significant retention in the skills.[13] Shahabe A et al based on the facts received from the study of healthcare interns from the university hospital concludes average awareness and below average knowledge of BLS despite having a good attitude toward BLS training and recommends that the BLS training program should not only be included in the university curriculum of all healthcare faculties but also be updated at regular intervals. [14]

As a future health care professional and the responsible citizen of the country, every medical student must be acquainted with BCLS training such that emergencies arising in our day to day life can effectively be managed. The training and experience enhance the knowledge and improves the ability of the student to manage medical emergencies there by, improves the community health. Medicos can form the role model to many people in the emergency and can also impart the much-required awareness regarding BCLS to all. This way we can effectively fulfil the responsibility rested on our shoulders. A formal BCLS refresher training is essential for retention of BCLS skills and to maintain competency in the technique. In community lay person should be encouraged to participate in such type of workshops. In future scientific laboratory should be established in all medical colleges for standardization of quality CPR.

Standardized training in BCLS shall be incorporated in all the medical and paramedical training programs. Emergency medicine has already been recognized by Medical Council of India as a Subspecialty for a decade. Its high time that spreading awareness and teaching the basic and comprehensive life support to the medical and paramedical team as well as teaching compression only life support or hands only CPR at the community level should be the goal of this new emerging branch.

\section{CONCLUSIONS}

Basic Life Support training is an absolute necessity for the medical students to face acute medical emergencies that they come across, even while pursuing their graduation. This also boosts the confidence of students and save many lives.

\section{Recommendations}

1. Health Awareness Camps on BLS training so that public health workers \& general population would be aware of BLS.

2. There is a need to conduct more workshops, there by students can improve their practical skills.

3. BLS courses should be introduced at the earliest possible time into the medical curriculum.

4. Skill Labs or Scientific Laboratories should be established in all medical colleges in future for building awareness and standardization of quality of CPR. 


\section{REFERENCES}

[1] Miller BF, Keane C. Encyclopedia and dictionary of medicine, nursing and allied health. $2^{\text {nd }}$ edn. Toronto: Saunders 1978: p. 878.

[2] Zaheer H, Haque Z. Awareness about BLS (CPR) among medical students: status and requirements. J Pak Med Assoc 2009;59(1):57-9.

[3] Neurman RW, Shuster M, Callaway CW, et al. Part 1: Executive summary: 2015 American Heart Association Guidelines Updates for CPR \& ECC. Circulation 2015;132(18 Suppl 2):S315-S67.

[4] Vaillancourt C, Stiell IG, Canadian Caediovascular Outcomes Research Team. Cardiac arrest care and emergency medical services in Canada. Can J Cardiol 2004;20(11):1081-90.

[5] Beck JD, Eke P, Heiss G, et al. Periodontal disease and coronary heart disease: a reappraisal of the exposure. Circulation 2005;112(1):19-24.

[6] Skinner DV, Camm AJ, Miles S. Cardiopulmonary resuscitation skills of preregistration house officers. $\mathrm{Br}$ Med J (Clin Res Ed) 1985;290(6481):1549-50.

[7] Casey WF. Cardiopulmonary resuscitation: a survey od standards among junior hospital doctors. J R Soc Med 1984;77(11):921-4.

[8] Ruesseler M, Weinlich M, Muller MP, et al. Simulation training improves ability to manage medical emergencies. Emerg Med J 2010;27(10):734-8.
[9] Chandrasekaran S, Kumar S, Bhat SA, et al. Awareness of basic life support among medical, dental, nursing student and doctors. Indian Journal of Anaesthesia 2010;54(2):121-6.

[10] Zaheer H, Haque Z, et al. Awareness about BLS (CPR) among medical students: status and requirements. J Pak Med Assoc 2009;59(1):57-9.

[11] Graham CA, Guest KA, Scollon D. Cardiopulmonary resuscitation. Paper 1: A survey of undergraduate training in UK medical schools. Journal of Accident and Emergency Medicine 1994;11(3):162-4.

[12] Abbas A, Bukhari SI, Ahmad F. Knowledge of first aid and basic life support amongst medical students: a comparison between trained and un-trained students. J Pak Med Assoc 2011;61(6):613-6.

[13] Pande S, Pande S, Parate V, et al. Evaluation of retention of knowledge and skills imparted to first-year medical students through basic life support training. Adv Physiol Educ 2014;38(1):42-5.

[14] Saquib SA, Al-Harthi HM, Khoshhal AA, et al. Knowledge and attitude about basic life support and emergency medical services amongst healthcare interns in university hospitals: a cross-sectional study. Article ID 9342892, Emergency Medicine International 2019;2019: p. 8. 